\title{
Teoria e Prática de IHC na Concepção do Sustenta Aí: Um Aplicativo para Promoção de Ações Sustentáveis
}

\author{
Douglas Henrique E. de O. Lima, José Vinnicius dos Santos Oliveira, Mirelly Naianny \\ Pereira da Silva, Yuska Paola Costa Aguiar \\ ${ }^{1}$ Departamento de Ciências Exatas - Universidade Federal da Paraíba Rua da Mangueira, \\ s/n, Companhia de Tecidos Rio Tinto 58.297-000 - Rio Tinto - PB - Brasil \\ \{douglas.emiliano, vinnicius.santos, mirelly.naianny, yuska\}@dcx.ufpb.br
}

Abstract. Approaches that combine theory and practice are useful for the education of students from Computer Science courses. A Challenge Project is adopted in the Human Computer Interaction discipline to cover the "Life Cycle of Interaction Design" content, including techniques, methods, and artifacts generation. The objective of each team is to propose a technological solution to increase comprehension and encourage sustainable actions in the everyday life of the community. The Sustenta Ai app was designed by a group of three students, based on the methodology that combined theory and practice, throughout the discipline. This article describes the applied methodology, the Sustenta Ai app, and the perspective of the participants (students and the professor) about the experience.

Resumo. A associação entre teoria e prática é recomendada na formação de discentes dos cursos de Computação. Para a disciplina de Interação Humano Computador um Projeto Desafio foi adotado para o conteúdo do "Ciclo de Vida do Design da Interação", incluindo técnicas, métodos e geração de artefatos. Os discentes tiveram como objetivo propor uma solução tecnológica para conscientizar e estimular ações sustentáveis no dia-a-dia. O aplicativo Sustenta Aí foi concebido por um grupo de 3 discentes, a partir da metodologia que aliou teoria e prática ao longo da disciplina. Este artigo descreve a metodologia aplicada, o app Sustenta Ai, e a perspectiva dos participantes (discentes e docente) sobre a experiência.

\section{Introdução}

A Sociedade Brasileira de Computação (SBC), em conformidade com as Diretrizes Curriculares Nacionais ${ }^{1}$, definiu em 2017 os Referenciais de Formação para os Cursos de Graduação em Computação [Zorzo et al., 2017]. Tal iniciativa tem por objetivo fornecer insumos para auxiliar coordenadores de curso de graduação na elaboração de projetos pedagógicos dos cursos na área de Computação. Sua utilidade também se revela para que docentes possam direcionar suas abordagens de ensino seguindo tais recomendações. Para tanto, o referido documento é composto por recomendações no tocante ao perfil esperado para o egresso de acordo com o objetivo geral do curso, sendo este decomposto em diferentes eixos de formação. Estes objetivam capacitar o egresso em competências genéricas e específicas, alcançadas a partir da exploração de conteúdos pré-definidos. Além disso, são indicadas metodologias de ensino que podem ser adotadas para potencializar os resultados pretendidos para com a formação dos estudantes.

\footnotetext{
${ }^{1}$ Resolução No 05/2016; MEC, 2016.
} 
Estes insumos foram analisados sob a ótica da realidade vivenciada na Universidade Federal da Paraíba, Campus IV, Rio Tinto, especificamente para os cursos de Licenciatura em Ciência da Computação (LCC) e Bacharelado em Sistemas de Informação (BSI). A análise foi realizada com o intuito de elaboração e adoção de uma abordagem metodológica de ensino em consonância com as recomendações da SBC para o componente curricular de Interação Humano-Computador ${ }^{2}$ (IHC). Segundo a SBC, tal disciplina é componente curricular para os eixos de formação 2 e 5 no curso de LCC (fundamentos da computação e tecnologias na educação) e 1 e 3 no curso de BSI (visão sistêmica e desenvolvimento de software para sistemas de informação).

Este componente curricular está presente nos projetos pedagógicos para os cursos supracitados sob a nomenclatura de Interface Homem-Máquina (03 créditos, carga horária de $45 \mathrm{~h})^{3}$ e Interface com o Usuário ${ }^{4}$ (04 créditos, carga horária de $60 \mathrm{~h}$ ). Vale destacar que parte da ementa definidas para as disciplinas de ambos os cursos é comum, e consiste em abordar "Metodologias, técnicas e ferramentas de concepção, projeto e implementação de sistemas interativos", assim como "Metodologias, técnicas e ferramentas de avaliação de interfaces".

Para ambos os cursos, considerando suas especificidades técnicas, a SBC indica como perfil esperado para os egressos a capacidade de responderem às demandas da sociedade a partir do uso da Computação como mediadora no processo de resolução de problemas, ou antecipação estratégica das necessidades da sociedade - seja no meio educacional e/ou empreendedor. Ainda de forma homogênea para os cursos, a SBC sugere a adoção de metodologias de ensino que priorizem a articulação entre teoria e prática, contemplando o desenvolvimento de atividades que priorizem o educar pela pesquisa, a autonomia, a curiosidade e a criatividade, favorecer o desenvolvimento de relações interpessoais, por meio da própria experiência do estudante. Destaca-se ainda, a técnica de Aprendizagem Baseada em Projetos (ABP) como uma possibilidade de abordagem a ser adotada. Esta é compreendida como uma estratégia transdisciplinar para aprendizagem, centrada no aluno e por meio da investigação, tendo em vista à produção de conhecimento individual e grupal [Souza, 2015]

Diante do exposto, este artigo apresenta o relato da experiência vivenciada no contexto da disciplina de IHC, ofertada para os cursos de graduação de LCC e BSI, para o semestre letivo 2019.2 na UFPB-Campus IV.

\section{Projeto Desafio sobre Sustentabilidade no ensino de IHC}

Para o período letivo 2019.2 a disciplina de IHC foi oferecida para os cursos de LCC e BSI, na UFPB-Campus IV, contemplando um total de 40 alunos matriculados, dos quais 36 cursaram a disciplina até o final. A predominância de alunos oriundos do curso de BSI foi uma realidade esperada, dado que este componente curricular é obrigatório, sendo optativo aos alunos de LCC. O perfil misto da turma não foi comprometido em relação aos objetivos específicos dos respectivos cursos, devido à similaridade de ementa.

$\mathrm{Na}$ busca por atender as recomendações da SBC, os conteúdos supracitados foram trabalhados considerando: (i) a conexão constante entre teoria relativa ao Ciclo de Vida do

\footnotetext{
${ }^{2}$ A nomenclatura Interação Humano-Computador (IHC) será adotada ao longo do texto para referenciar os termos Interface Homem-Máquina e Interface com Usuário, pela atualidade e maior abrangência desta.

${ }^{3} \mathrm{http} / / / \mathrm{www} . c \mathrm{cae} . u f \mathrm{pb} . \mathrm{br} / \mathrm{lcc} / \mathrm{contents/documentos/ppc-lcc-atualizado-em-2012.pdf}$

${ }^{4}$ https://si.dcx.ufpb.br/detalhes-das-disciplinas/\#interface
} 
Design da Interação - Rogers, Sharp, Preece (2013) e prática da aplicação dos métodos para execução das etapas do mesmo; (ii) dentro do contexto da execução de um Projeto Desafio, a partir do qual os alunos deveriam trabalhar em equipe para propor uma solução de tecnologia para resolução de um problema real da nossa sociedade; e (iii) em um contexto de favorecimento à criatividade e a autonomia dos alunos, por meio de sua própria experiência, durante todo o processo.

O Ciclo de Vida do Design da Interação é iterativo e incremental, contemplando, de forma abstrata e cíclica as seguintes etapas: o início do processo se dá pela Identificação de Necessidades e Estabelecimento de Requisitos, seguida pelo (re)Design, que permite a elaboração da Construção de uma Versão Interativa da solução que é Validada e em seguida refinada a partir de nova rodada do ciclo (Figura 1).

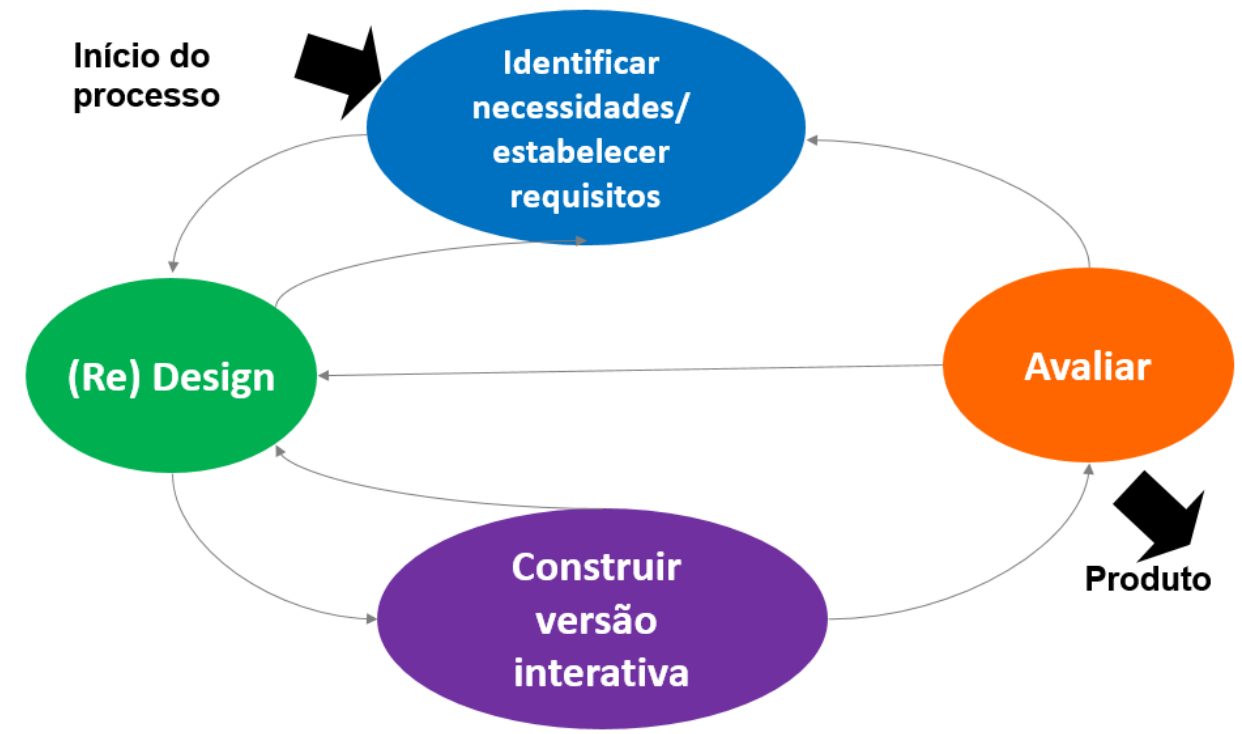

Figura 1: Ciclo de Vida do Design de Interação

Para cada uma destas etapas, os conteúdos teóricos foram abordados em sala com apresentação de exemplos para os vários métodos e técnicas que puderam ser empregados pelos alunos na execução do Projeto Desafio. Tendo em vistas a necessidade de não apenas apresentar os conteúdos teóricos aos alunos, mas também fazê-los refletir sobre a adequação dos métodos e técnicas a aplicar, para o Projeto Desafio os alunos tiveram a liberdade sobre a escolha de método/técnica, sendo solicitado que apresentassem uma justificativa que permitisse identificar seu senso crítico e de coerência na tomada de decisão no tocante à fase de desenvolvimento da solução, ao nível de conhecimento exigido para empregar o método, ao tempo e recursos disponíveis, etc.. Todas as etapas do Ciclo de Vida do Design da Interação puderam ser vivenciadas pelos alunos no contexto do Projeto Desafio, havendo momentos específicos para apresentação e discussão dos resultados parciais em sala de aula, com a participação de todos os alunos, sendo mediada pelo professor.

Vale destacar que um conceito chave em IHC é relativo à compreensão que os projetistas devem assumir sobre o usuário da solução em desenvolvimento, assim como sobre a participação deste usuário no processo tomada de decisão sobre o projeto em si. Dentro deste conceito tem-se o Design Centrado no Usuário - Abras et al., (2004) e o Design Participativo - Sanders (2002) como duas abordagens que devem ser essencialmente exploradas durante a disciplina. Durante a execução do Projeto Desafio os alunos foram 
estimulados à aplicarem estas abordagens em diferentes momentos do Ciclo de Vida da Interação.

O Projeto Desafio teve como slogan a ideia de que "Todo lixo é um erro de design", deixando em evidência o tema central a ser trabalhado: Sustentabilidade. O objetivo definido foi de: elaborar uma solução para apoiar a conscientização e a adoção de práticas sustentáveis no "tratamento" de lixo - um problema socialmente conhecido no Brasil. A solução deveria ajudar uma ou mais esferas específicas da sociedade a lidar com o problema do lixo não tratado de forma sustentável, promover a conscientização das pessoas sobre o problema e sobre a importância de minimizar a produção de lixo a partir das práticas sustentáveis. Deixou-se claro que era esperado que a solução explorasse diferentes paradigmas de IHC.

Para promover uma maior familiarização dos alunos com o tema da Sustentabilidade, um Professor Doutor em Design Sustentável foi convidado para fazer uma intervenção com a turma para explanar sobre os "Princípios da sustentabilidade e a problemática do lixo sob a perspectiva dos designers". Em seguida, os alunos foram motivados a realizarem pesquisa sobre o tema, assim como buscar por soluções existentes que pudessem ser fontes inspiradoras no processo criativo de proposição de suas soluções. Os alunos tiveram liberdade para escolher o tema específico a explorar dentro da imensidão de possibilidades relacionadas à Sustentabilidade. A correlação entre os conteúdos ministrados e as atividades do projeto desafio estão organizadas de acordo com as etapas do Ciclo de vida do Design da Interação (Quadro 1).

\section{Quadro 1: Mapeamento entre conteúdos teóricos e atividades práticas}

Identificação de Necessidades e Estabelecimento de Requisitos

- Teoria: Técnicas de coleta de dados (Estudo da documentação; Análise comparativa de propostas similares; Aplicação de questionários e entrevistas; Grupo Focal e Observação de Uso)

- Prática: Adotar 2 ou 3 técnicas de elicitação de requisitos e responder a um conjunto de questões sobre o usuário, seu contexto de uso e atividades a executar na solução proposta

(re)Design

- Teoria: Modelagem conceitual da interface: Personas e Modelo Hierárquico da tarefa

- Prática: Aproveitar os dados coletados na atividade anterior e definir 3 personas para representação dos potenciais usuários e definir o Mínimo Produto Viável (MVP)

Construção de uma versão interativa

- Teoria: Card sorting, Mapa de navegação e Prototipagem (baixa, média e alta-fidelidade)

- Prática: Aplicação do card sorting para o MVP e realizar prototipagem de baixa-fidelidade incluindo mapa de navegação $\left(1^{\circ}\right.$ ciclo $)$ e de média-fidelidade $\left(2^{\circ}\right.$ ciclo $)$

Validação

- $\underline{\text { Teoria: }}$ Avaliação com especialista e com usuários (avaliações rápidas e sujas, percurso cognitivo, heurísticas, inspeção, estudo de campo, grupo focal, verbalização de procedimentos, entrevista/questionário e teste de usabilidade)

- Prática: Aplicar 2 ou 3 técnicas de avaliação para validação dos protótipos de baixa e média fidelidade $\left(1^{\circ}\right.$ e $2^{\circ}$ ciclo, respectivamente)

Como entrega, além dos artefatos gerados ao longo da execução do projeto, os alunos foram instruídos a produzir e disponibilizar (i) a solução em nível de protótipo de média/altafidelidade, que permita simular a execução da ação/tarefa mais relevante da solução; (ii) um vídeo demonstrativo de até 2 minutos de duração apresentando a solução e (iii) um relatório de 3 páginas (modelo SBC) com a descrição da solução. O conjunto de entregas permite que os alunos explorem diferentes formas e linguagens para comunicar os resultados de um projeto que consiste em uma solução destinada ao grande público. 
Os alunos se organizaram de forma livre (por afinidade ou conveniência) em grupos contendo entre 3 e 5 integrantes. Ao todo 9 grupos foram formados e 9 soluções foram elaboradas e finalizadas no contexto da disciplina. Neste artigo o percurso e os resultados alcançados por uma das equipes serão explanados de forma demonstrativa. A solução proposta pelo grupo de 3 alunos de LCC chama-se Sustenta Aí e está descrita detalhadamente na Seção 3. A escolha por este projeto se deu pelo rigor técnico e qualidade dos resultados alcançados, assim como pelo interesse declarado dos integrantes da equipe em ampliar o relatório entregue para consolidação deste artigo ${ }^{5}$.

\section{Aplicando o Projeto de Design da Interação para o Sustenta aí}

O Sustenta Aí é uma aplicação mobile cujo propósito é auxiliar no processo de educação ambiental, mais especificamente para promover a adoção de práticas sustentáveis no dia a dia da população, como a separação de material para descarte que propicie a reciclagem. $\mathrm{O}$ motivador para o desenvolvimento desta solução está alinhado com a realidade atual, na qual o movimento sustentável vem tomando espaço considerável na sociedade moderna, deixando o anonimato em direção à um protagonismo cada vez mais notório. Porém, sabendo-se que a caminhada rumo à difusão necessária para equilibrar a balança da degradação ainda é longa. Logo, se faz necessário facilitar a adoção de medidas sustentáveis para despertar na sociedade a vontade de introduzir sustentabilidade como um hábito. Além da recente disseminação de informações sobre o tema, uma familiarização sobre o ciclo de vida do produto e a sustentabilidade - Finkbeiner et al., (2010) foi possível a partir da palestra proferida no escopo da disciplina.

O Projeto Desafio executado para o Sustenta Aí foi dirigido ao alinhamento implícito entre o ciclo de vida do design da interação e os conceitos relativos ao Design Thinking [Brown e Wyatt, 2010]. O conhecimento sobre esta abordagem foi possível a partir da palestra sobre Inovação e Design Thinking, proferida por um professor pesquisador convidado para participar na disciplina de IHC. O Design Thinking é organizado em: entender, explorar e materializar uma solução - cuja correspondência pode ser associada à Identificação de Necessidades e Estabelecimento de Requisitos, (re)Design e Construção de uma versão interativa, respectivamente [Berger, 2011].

A definição do foco e do escopo iniciais para o Sustenta Aí foi resultante da primeira etapa do ciclo de vida do design da interação: Identificação de Necessidades $e$ Estabelecimento de Requisitos - considerando uma exploração inicial ${ }^{6}$. A execução prática relativa às técnicas de coletas de dados se deu a partir da análise de notícias e artigos sobre "práticas de sustentabilidade", assim como da análise de exploratória de produtos similares.

Esta exploração inicial serviu como norte para refinamento das ideias iniciais de forma a estabelecer três eixos como fundamentos para as decisões de projeto para o Sustenta Aí, a saber: (i) Redes Sociais: a aplicação deve ser utilizada para reunir pessoas com ideais em comum para promoção de relacionamento virtual, troca de experiências e principalmente o estímulo para a inserção de práticas sustentáveis no dia a dia dos integrantes desta comunidade; (ii) Gamificação: adotar uma estratégia de usar elementos comuns dos jogos em situações que não se restringem ao entretenimento. A adoção de elementos de jogos (níveis, recompensas e badges) para tornar a aplicação mais atrativa para os usuários,

\footnotetext{
5 Todas as equipes foram questionadas sobre o interesse em ampliar a entrega no formato de artigo.

${ }^{6}$ Outras práticas associadas à esta etapa estão descritas na subseção 3.1.
} 
promovendo maior engajamento durante sua utilização - da Cunha, Gasparini e Berkenbrock, (2013) elemento chave em uma rede social. Além disso, segundo Oliveira, Figueiredo e Souza (2019) gamificar experiências educacionais têm demonstrado resultados positivos; (iii) Aplicativos comerciais: com vistas a envolver estabelecimentos comerciantes locais enquanto centros de coleta para o descarte dos produtos recicláveis, atuando, ainda, no processo de gamificação fornecendo recompensas à população participante. $O$ estabelecimento que adere à causa ganha visibilidade enquanto empresa parceira na luta em prol da sustentabilidade e do bem estar comum da população.

A combinação entre os três eixos supracitados permitiu que a aplicação Sustenta Aí integre os modelos como àqueles adotados em programas de relacionamento (ex.: Dotz e Smiles) a partir dos quais os participantes ao realizarem o descarte de produtos recicláveis geram pontuação para trocar por prêmios. Considera, ainda o modelo de geolocalização (ex.: Cittamobi e Pokémon Go) para que o participante encontre os pontos de descarte mais próximo à sua localização, assim como permite que os participantes comparem seus perfis no formato de rankings (ex.: Duolingo). Nas subseções seguintes são descritas as atividades realizadas e os artefatos gerados para o Sustenta aí, de forma a exemplificar a instanciação para a metodologia adotada na disciplina de IHC (Quadro 1).

\subsection{Identificação de necessidade e Estabelecimento de Requisitos}

A técnicas adotada para coleta de dados junto aos potenciais usuários do Sustenta Aí foi a aplicação de questionário online. Este foi organizado em três seções: sondagem do perfil (informações pessoais tais como: faixa etária, escolaridade e gênero), sondagem sobre o conhecimento dos respondentes sobre práticas sustentáveis, e sondagem sobre os principais problemas enfrentados pelos respondentes para adesão e manutenção de hábitos sustentáveis na sua rotina. O questionário foi divulgado via WhatsApp e Instagram, no período de 05 a 07 de março de 2020 e obteve um total de vinte participantes (anonimato adotado para preservar privacidade). Esta técnica foi selecionada pois permite uma fácil aquisição de dados e uma exportação simplificada que permitiu analisar os dados obtidos de forma clara e concisa. Como resultado inicial da análise dos dados coletados, foi possível refinar as ideias iniciais para a solução proposta ao responder às questões direcionadas indicadas como parte desta atividade, a saber:

- Sobre os potenciais usuários para a solução proposta: (i) de quem estamos falando? (ii) o que é familiar para eles (no contexto da sustentabilidade e reciclagem de produtos)? (iii) quais são suas necessidades e desejos? (iv) o que eles pensam a respeito deste projeto;

- Sobre as atividades mediadas pela solução: (v) quais são os objetivos dos usuários no uso desta solução? (vi) vocês poderiam descrever algumas "coisas" que os usuários podem fazer com a solução proposta? (vii) o que motiva os usuários (como esta informação pode agregar valor ao projeto); e

- Sobre o contexto no qual o projeto da solução proposta se encontra: (viii) quais os colaboradores deste projeto? (ix) quais são os possíveis "concorrentes" deste projeto? (x) quais as tecnologias ou dispositivos envolvidos? e, (xi) qual é o ambiente tecnológico necessário para dar suporte a este projeto?

\section{2. (Re)Design}

A realização desta etapa teve por objetivo a obtenção de dois resultados: definição de 3 personas que representam perfis dos potenciais usuários da aplicação Sustenta Aí; e a 
definição do Mínimo Produto Viável (MVP), ou seja, a identificação das funcionalidades prioritárias para a disponibilização de uma versão inicial da aplicação. Para tanto, foi realizada a análise dos dados obtidos na fase anterior (Seção 3.1), assim como modelou-se as funcionalidades pretendidas para a aplicação de forma hierárquica, com base nas tarefas (atividades) que os usuários poderiam realizada via aplicação.

As três personas ${ }^{7}$ obtidas se caracterizam como: (i) Helena Buarque, uma professora universitária de 40 anos que, preocupada com seus filhos e alunos, passou a incluir práticas sustentáveis no seu dia-a-dia. No entanto, Helena sente bastante dificuldade na separação do lixo no condomínio onde vive, pois os responsáveis não oferecem suporte à coleta e separação de materiais recicláveis adequadamente; (ii) Marcus Ribeiro, um jovem estudante universitário e ativista no quesito "Direito dos Animais". Pensando neles, Marcus repudia todo e qualquer ato de degradação do meio ambiente e sempre carrega consigo seu lixo quando não há lixeiras por perto, pois tem ciência do mal que o lixo pode causar aos animais. Sua maior frustração é com os governantes da sua cidade, segundo Marcus, a cidade não possui sistemas de coleta seletiva de lixo. Sempre que tem oportunidade, ele orienta amigos e familiares a separarem corretamente seus lixos; (iii) Roberta Monteiro, uma Engenheira de Software de 39 anos é usuária assídua de programas de relacionamento que, aproveitando as facilidades dos mesmos pode realizar suas viagens enquanto cursava seu mestrado na Europa. Roberta percebe a diferença como países ditos desenvolvidos tratam seu lixo. Por conta disso, passou a adotar hábitos sustentáveis em sua vida, como: uso de ecobags, separação de materiais recicláveis do lixo e seu descarte para cooperativas de reciclagem.

O MVP identificado para a versão inicial do Sustenta Aí consiste na entrega o conjunto das seguintes funcionalidades: Realizar login e logout; Cadastrar e alterar informações pessoais (nome, telefone, e-mail, CPF, data de nascimento e endereço); Efetuar coleta/descarte de materiais nos locais definidos no mapa; Cadastrar uma nova coleta/descarte de material no mapa; Trocar os pontos obtidos por brindes nos estabelecimentos parceiros; Seguir perfis de usuários que realizam descartes frequentemente; favoritar e desfavoritar um perfil; Buscar um perfil dentre a lista de favoritos.

\subsection{Construção de uma versão interativa e Validação}

Como forma de entender e elaborar o fluxo de navegação adequado para o Sustenta Aí e seus usuários, utilizou-se o método de Card sorting. Este é utilizado para organizar as funções de uma aplicação, permitindo visualizar sua estrutura hierárquica básica e identificar similaridades de funcionalidades a serem agrupadas. Os recursos utilizados foram: cartolina, post-its e canetas coloridas. As funcionalidades tiveram seus nomes escritos nos post-its, que foram colados na cartolina de forma hierárquica, respeitando o princípio da proximidade e relação entre as funcionalidades, sendo estas conectadas a partir de linhas. Como resultado desta atividade foi estabelecido, ainda, o mapa de navegação da aplicação.

Posteriormente, foi realizada a construção do protótipo de baixa-fidelidade. Este recurso, geralmente construído com lápis e papel, auxilia como facilitador na comunicação entre os usuários e os projetistas/desenvolvedores e os potenciais usuários da aplicação. Consiste em uma primeira concretização visual das funcionalidades estabelecidas no MVP. Logo, o protótipo gerado permitiu visualizar o fluxo de navegação entre as telas da aplicação, sendo possível conhecer os caminhos pelos quais os usuários podem interagir com a interface do Sustenta Aí (Figura 2a). Em seguida, visando facilitar o processo de validação, os

\footnotetext{
${ }^{7}$ A representação gráfica das personas está disponível em: https://imgur.com/a/dcGNgiU
} 
desenhos do protótipo de baixa-fidelidade foram fotografados e utilizados no aplicativo Marvel para promover a transição entre as telas simulando o uso real da aplicação. Surge o protótipo de média fidelidade (Figura 2b), onde os desenhos se tornaram interativos, com navegação e cliques.

Este protótipo foi apresentado em sala de aula e alguns testes foram realizados, de forma exploratória, com alunos da turma que se dispuseram a participar. Recebidos os feedbacks do professor e alunos participantes da avaliação, o próximo passo foi o desenvolvimento de um protótipo de média-fidelidade, mais refinado em termos de look-andfeel (Figura 2c). Para tanto, foi utilizado o software de prototipagem profissional Figma para gerar esta versão refinada do MVP. Após análises e comparações entre diversos softwares existentes (Adobe XD, Sketch e Framer), o Figma foi escolhido por ser fácil de aprender e usar e por dispor de inúmeros recursos, inclusive a colaboração simultânea entre diversos designers no mesmo documento.

Para criação do protótipo no Figma as seguintes etapas foram realizadas: (i) definição de um Style Guide como ponto de partida flexível para definição de um estilo visual a ser aplicado de forma responsiva (site, desktop e mobile) [Nunes, 2017]; (ii) criação de um Design System baseado na abordagem do Atomic Design, metodologia composta por cinco estágios distintos trabalhando juntos para criar sistemas de design de interface de uma maneira mais deliberada e hierárquica [Frost, 2016].

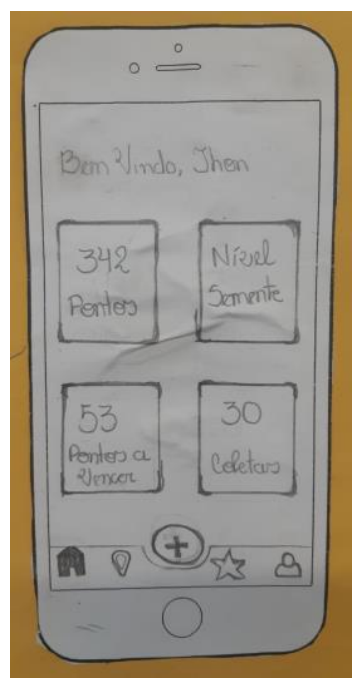

$\mathrm{a}$

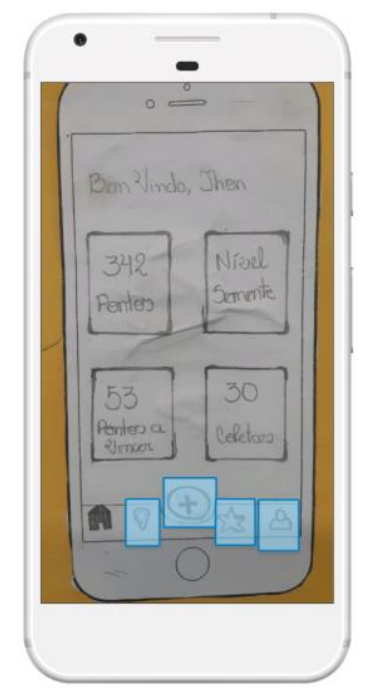

b

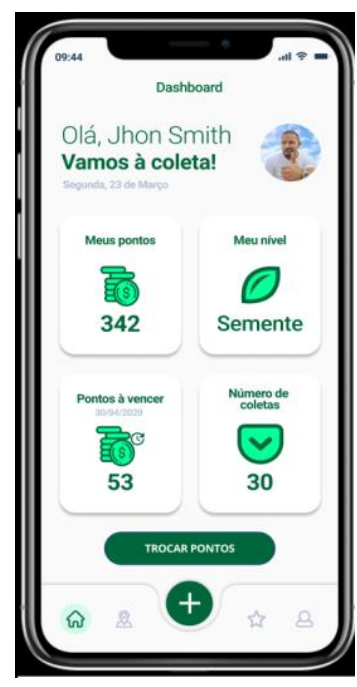

$\mathrm{c}$

Figura 2: Protótipos Sustenta Aí em a) Baixa-Fidelidade (Lápis e Papel), b) Média Fidelidade (Marvel app) e c) Média-Fidelidade (Figma)

\subsection{Sustenta Aí: Solução Proposta}

A dinâmica do Sustenta Aí funciona recompensando quem coleta e quem dispõe material para reciclagem. Os descartes/coletas geram pontos a serem trocados por prêmios dispostos em uma loja virtual do próprio aplicativo. Além disso, as experiências de descarte/coleta sustentável geram um ranking semanal daqueles com mais ações sustentáveis. Ambas as estratégias são motivadores para o uso do aplicativo e adoção de práticas sustentáveis. O protótipo de média-fidelidade resultante pode ser acessado a partir do link: 
http://tiny.cc/4xzrqz, assim como pode ser possível assistir ao vídeo demonstrativo de uso no YouTube: https://youtu.be/UEm16FVWpHo.

O menu da aplicação contém as cinco funcionalidades chaves: home onde as informações principais são dispostas em cards; o mapa de descarte/coleta para todos os locais disponíveis na região; os perfis favoritos que permitem um rápido acesso a usuários onde existe oferta recorrente; e o perfil de usuário que possui informações pessoais do usuário e o botão para criação de nova coleta. A tela principal é um dashboard, onde as informações mais importantes são apresentadas. Com a utilização da abordagem da gamificação o dashboard se tornou mais dinâmico e intuitivo com a inserção de elementos de jogos, como o sistema que mostra o nível atual do usuário, sua posição no ranking, bem como os badges para ilustrar suas conquistas (Figura 2c).

Além das informações supracitadas, a tela principal conta com um botão "Call to Action" que direciona o usuário à tela da troca de pontos. Ou seja, quanto o usuário consegue juntar pontos suficientes, então ele entra no aplicativo e analisa quais produtos pode obter. Logo, pode decidir por trocar seus pontos por uma camiseta cuja matéria prima é oriunda de garrafas pet recicladas, produzidas por uma empresa que aproveita o material depositado no posto de coleta vinculado ao aplicativo. $\mathrm{O}$ usuário além de contribuir evitando que garrafas pet (que demoram centenas de anos para se decompor na natureza) tivessem um destino incorreto, conseguiu obter material de fontes confiáveis e que trazem a certeza de que a luta para salvar o meio ambiente é mais do que válida, é uma garantia de um futuro que fica cada vez mais próximo.

\section{Lições Aprendidas e Considerações Finais}

A solução proposta para o Sustenta Aí integra a maior parte de conceitos teóricos vistos na disciplina de IHC, resultando em um protótipo navegável contendo o MVP definido enquanto escopo do projeto de design da interação. Além dos conteúdos teóricos vistos em sala e as respectivas atividades práticas desenvolvidas enquanto projeto da disciplina (seções 2 e 3 , respectivamente), a solução proposta foi construída utilizando como alicerce as 10 Heurísticas de Nielsen - Nielsen, (1995), ou seja os 10 princípios mais gerais para o design de interação, conteúdo também trabalhado em sala.

Diante da vivência do professor proponente do Projeto Desafio, percebeu-se o envolvimento dos alunos na execução de suas soluções, com alinhamento explícito entre teoria e prática. Ainda, acompanhou-se a evolução e maturação das ideias iniciais apresentadas pelos alunos em protótipos evolutivos em termos de fidelidade (look-and-feel). Nesta experiência percebeu-se que os alunos saíram de uma percepção exclusiva sobre o cliente da aplicação, para compreender o papel e a importância dos usuários da aplicação um dos desafios enfrentados nas disciplinas de IHC.

Sob a perspectiva dos alunos que integraram a equipe que concebeu o Sustenta Aí, alguns pontos foram evidenciados e aqueles apontados como passíveis de melhoria devem ser considerados no refinamento da proposição para uma próxima turma. Seguem os relatos:

- "A disciplina de IHC como parte do currículo dos cursos de TI, insere mais opções de carreiras para o estudante, que de alguma maneira não se identifica com programação e manutenção de computadores. Durante a oferta da disciplina foi possivel experienciar na prática os desafios enfrentados por um profissional que lida 
diretamente com a experiência do usuário no processo de desenvolvimento de softwares";

- "O tema de sustentabilidade foi de bastante relevância, pois precisamos despertar sobre novos meios para lidar com este problema";

- "Como as aulas aconteciam apenas uma vez por semana e com o acúmulo de afazeres de outras disciplinas, houve uma sensação de sobrecarga. Todavia, a pressão psicológica exercida, justamente pela falta de tempo, fez com que a gente fosse inserida, mesmo que de forma abstrata, num cenário real de ambiente de trabalho".

\section{Referências}

Abras, C., Maloney-krichmar, D., Preece, J., et al. (2004) "User-centered design." Bainbridge, W. Encyclopedia of Human-Computer Interaction. Thousand Oaks: Sage Publications, vol. 37, no 4, p. 445-456.

Berger, A. (2011) "Design thinking for search user interface design". In: Proceedings of EuroHCIR2011, p. 1-4.

Brown, T., Wyatt, J. (2010) "Design thinking for social innovation". Development Outreach, vol. 12 , no 1 , p. 29-43.

da Cunha, L. F.; Gasparini, I.; Berkenbrock, C. D. M. (2013) "Investigando o uso de gamificação para aumentar o engajamento em sistemas colaborativos”. In: Anais do V Workshop sobre Aspectos da Interação Humano-Computador para a Web Social. SBC. p. 28-33.

Finkbeiner, M., Schau, E. M., Lehmann, A., et al. (2010) "Towards life cycle sustainability assessment." Sustainability, vol. 2, no 10, p. 3309-3322.

Frost, B. (2016). “Atomic design. Pittsburgh".

Nielsen, J. (1995). “10 usability heuristics for user interface design”. Nielsen Norman Group, v. 1, n. 1 .

Nunes, Mark Klaus (2020). "UI Style Guide - Definindo a Abordagem Visual”, Medium.

Oliveira, J. V. S.; Figueiredo, L. M. R.; Souza, F. L. (2019). “Oficina de Apoio a Resolução de Problemas e Lógica Computacional Utilizando a Computação Desplugada e Gamificação". In: Congresso sobre Tecnologias na Educação (CTRL+E), 4, Recife, dec. 2019 . p. 497-503.

Sanders, E. B.-N. (2002). "From user-centered to participatory design approaches". In : Design and the social sciences. CRC Press. p. 18-25.

Souza, S. C. de. (2015). "Aprendizagem Baseada em Problemas (ABP): Um Método Transdisciplinar de Aprendizagem para Ensino Educativo".

Rogers, Y., Sharp, H., Et Preece, J. (2013). "Design de Interação". Bookman Editora.

Zorzo, A. F.; N., D.; Matos, E.; Steinmacher, I.; Leite, J.; Araujo, R. M.; Correia, R.; Martins, S. (2017). "Referenciais de Formação para os Cursos de Graduação em Computação". Sociedade Brasileira de Computação (SBC). 153p. 